from their description of disaster, which included the cause, category, characteristic, impact and type of disaster. $36.4 \%$ of students were certain that disaster would happen again in China. A further $50.9 \%$ felt disaster was likely. Flood and earthquake were considered the most likely future disasters. $71 \%$ of nursing students strongly agreed that being prepared for disaster was important. The main reasons were better preparedness could decrease the damage to property and the incidence of death and injury. However, the level of understanding of the effects of disaster and the exposure of students to education about disaster health response was limited.

Conclusion: Knowledge and skills for disaster preparedness of nursing students should be strengthened in the medical university.

Prehosp Disaster Med 2011;26(Suppl. 1):s109-s110

doi:10.1017/S1049023X11003669

(P1-35) Adapting a Humanitarian Organization for Disaster Response, Operation Smile in Haiti, 2010

R. Zoraster, ${ }^{1}$ M. Beers, ${ }^{2}$ T. Crabtree ${ }^{1}$

1. Honolulu, United States of America

2. Operation Smile, Norfolk, United States of America

Background: Operation Smile International (OSI) is a NonGovernment Organization (NGO) with experience providing surgical care throughout the world. OSI has vast logistical capacity, skilled and credential providers, and international relationships. Disaster response had been considered by OSI in the past, but never initiated. However, the magnitude of the Haiti disaster, coupled with request from Haitian OS Partners led to the initial disaster response of the OSI organization.

Discussion and Observations: This presentation will: (1) Describe the considerations and rationale that led OSI to this intervention. (2) Discuss the process of developing a disaster response within a relatively short period of time. (3) The response itself, and (4) Present how the lessons learned will be adapted to future OSI capacity and planning.

Prehosp Disaster Med 2011;26(Suppl. 1):s110

doi:10.1017/S1049023X11003670

\section{(P1-36) Mass Casualty Incident Awareness of Remote Location Staff in Western Australia \\ R. Goswami \\ Western Operations, Perth, Australia}

Introduction: The state of Western Australia has a remote population spread throughout an immense area. Remote health and retrieval is strained on a day-to-day basis, let alone in mass casualty incidents (MCIs). Anecdotally, remote medical staff has minimal training in MCI response. There is no research into how aware these staff is on principles of MCI response.

Methodology: An online survey was devised to ascertain the awareness and knowledge of medical staff most likely to be involved in a disaster. Demographic as well as questions in scenario format were disseminated to rural general practitioners (GPs), nurses and paramedics. Data was collected over a 4-month period.

Results: 117 surveys were completed online. Analysis revealed an astute awareness of resources and environment in a potential
MCI but triaging was poor and complex decision-making results were equivocal. Trained respondents handled scenarios better than experienced (MCI involvement or planning) respondents.

Conclusions: In general, remote medical staff is aware of only certain MCI principles. Further training is warranted. Voluntary feedback from these staff also strongly corroborated this view.

Prehosp Disaster Med 2011;26(Suppl. 1):s110

doi:10.1017/S1049023X11003682

(P1-37) Over and Undertriage in Simulation Exercises E. Styrwoldt

Department Of Education, Stockholm, Sweden

Over and undertriage in simulation exercises Introduction The first healthcare personnel arriving at the scene of an accident or major incident is often an ambulance crew. It is therefore of importance that they are familiar with, and can practice triage during situations were there is a lack of resources. Overtriage, when a casualty is given a priority higher than motivated, may lead to inadequate use of resources, while undertriage can be seen as a risk for medical errors. There is a consensus that up to $50 \%$ overtriage is accepted in order to have an undertriage, which is less than 5\%. The aim of this study was to increase knowledge regarding prehospital personnel's triage during standardized simulation exercises.

Material and Method: 76 standardized simulation exercises where the triage of casualties was evaluated. The exercises were part of a training program for medical command and control at scene. The students trained were all professional ambulance crew. The scenario was a fire at a football stand with 50 causalities. All in all 3800 (76 x 50) triages were performed. The simulation system used was Emergo Train System. Prior to the exercises an expert group had triaged the causalities according to the MIMMS system (sieve). Of the 50 patients 15 were triaged as $\mathrm{T} 1$ by the expert group and the rest were not.

Results: Of the 3800 triages $37 \%(n=410)$ were classified as undertriage and $13 \%(n=134)$ as overtriage. The most frequently undertriage casualties had an airway and/or breathing problem that were not observed. The most frequently overtriage casualties had a burn injury involving 30\% of body surface area or unconscious casualties.

Conclusions: Triage in this simulation setting did not meet acceptable standards. More triage training for ambulance crew may improve outcome. More studies are needed regarding simulation exercises as a tool for evaluating results of triage.

Prebosp Disaster Med 2011;26(Suppl. 1):s110

doi:10.1017/S1049023X11003694

(P1-38) Emergency Department Preparedness for Training Management Plan towards Mass Casualty Incidents

W.L. Chan

Emergency Medicine, Singapore, Singapore

It is important to equip emergency department (ED) staff with skills to manage mass casualty incidents (MCI) as disasters 